\title{
Caractérisations physico-chimiques et bactériologiques des eaux des stations aquacoles de Layo et de Jacqueville (Lagune Ebrié, Côte d'Ivoire)
}

\author{
Aubin Cyrille TOULE ${ }^{1,2 *}$, Ama Antoinette ADINGRA², Nadège KOUADIO-N'GBESSO², \\ Ollo KAMBIRE ${ }^{3}$, Rose KOFFI-NEVRY ${ }^{1}$ et Marina KOUSSEMON ${ }^{1}$ \\ ${ }^{1}$ Laboratoire de Biotechnologie et Microbiologie des Aliments, UFR des Sciences et Technologies des \\ Aliments, Université Nangui Abrogoua, 02 BP 801 Abidjan 02, Côte d'Ivoire. \\ ${ }^{2}$ Laboratoire de Microbiologies, Centre de Recherches Océanologiques, BP V18 Abidjan, Côte d'Ivoire. \\ ${ }^{3}$ Département de Biochimie et des Sciences des Aliments, Université de Péléforo Gon Coulibaly, BP 1328 \\ Korhogo, Côte d'Ivoire. \\ *Auteur correspondant,E-mail : toulenco@gmail.com ; toulenco@yahoo.fr ; Tel : (+225) 08453887.
}

\section{RESUME}

Les eaux des stations aquacoles et des milieux lagunaires sont susceptibles de contenir de grandes quantités de polluants organiques et de véhiculer une diversité d'agents pathogènes (virus, bactéries, parasites) pour l'homme et les espèces aquatiques. La préservation de ces écosystèmes nécessite donc une meilleure connaissance de leurs caractéristiques. Cette étude a été menée afin de déterminer la qualité de l'eau des stations aquacoles de Layo et de Jacqueville implantées sur les rives de la lagune Ebrié. Au total, 48 échantillons d'eau provenant de 8 sites ont été analysés de mars à octobre 2015. Les paramètres physicochimiques ont été mesurés in situ et analysés selon les méthodes conventionnelles de la chimie. Le dénombrement de coliformes fécaux et de Vibrio a été effectué selon la technique des membranes filtrantes. Le $\mathrm{pH}$ varie de 5,7 à 7,6 , la température de 28,2 à $30,1^{\circ} \mathrm{C}$, la salinité de 0,1 à $6,52 \%$, la conductivité de 0,23 à $5,84 \mathrm{mS} / \mathrm{cm}$ et l'oxygène dissous de 2,43 à $7,76 \mathrm{mg} / \mathrm{l}$. Les concentrations des solides totaux dissous varient de 0,18 à 10,21 mg/l et celles des matières en suspension de 4,3 à 57,3 mg/l. Les teneurs de nitrate et de nitrite sont respectivement de 0,06 à $1,75 \mathrm{mg} / \mathrm{l}$ et de 0,002 à $0,038 \mathrm{mg} / \mathrm{l}$. Les charges de coliformes fécaux varient de 3,21 à 4,29 $\log$ UFC/100 ml et celles de Vibrio de 3,3 à 4,26 $\log$ UFC/100 ml. Cette étude indique une forte contamination bactérienne des eaux et des concentrations de nitrate et nitrite dans les limites autorisées par l'OMS.

(C) 2017 International Formulae Group. All rights reserved.

Mots clés : Vibrio, coliformes, paramètres physico-chimiques, stations aquacoles, lagune Ebrié.

\section{Physicochemical and bacteriological characterization of water from the aquaculture stations of Layo and Jacqueville (Ebrié lagoon, Ivory Coast)}

\begin{abstract}
Waters of aquaculture stations and the lagoon environments are likely to contain large quantities of organic pollutants and to convey a diversity of pathogens (viruses, bacteria, parasites) for human and aquatic organisms. The preservation of these ecosystems therefore requires a better knowledge of their characteristics. This study was conducted to determine the water quality of aquaculture stations of Layo and Jacqueville
\end{abstract}


located on the shores of the lagoon Ebrié. In total, 48 samples of water from 8 sites have been analyzed from march to october 2015. The physico-chemical parameters were measured in situ and analyzed according to the conventional methods of chemistry. The fecal coliforms and Vibrio counts have been carried out according to the technique of membrane filtration. The $\mathrm{pH}$ ranges from 5.7 to 7.6 , the temperature from 28.2 to $30.1{ }^{\circ} \mathrm{C}$, the salinity from 0.1 to $6.52 \%$, the conductivity from 0.23 to $5.84 \mathrm{mS} / \mathrm{cm}$ and dissolved oxygen from 2.43 to 7.76 $\mathrm{mg} / \mathrm{l}$. The concentrations of total dissolved solids vary from 0.18 to $10.21 \mathrm{mg} / \mathrm{l}$ and those of suspended solids from 4.3 to $57.3 \mathrm{mg} / \mathrm{l}$. Nitrate and nitrite contents range respectively from 0.06 to $1.75 \mathrm{mg} / \mathrm{l}$ and from 0.002 to $0.038 \mathrm{mg} / \mathrm{l}$. fecal coliforms loads vary from 3.21 to $4.29 \mathrm{log}$ CFU/100 ml and Vibrio from 3.3 to $4.26 \mathrm{log}$ CFU/100 ml. This study indicates a high bacterial contamination of water and nitrate and nitrite levels are within the limits authorized by WHO.

(C) 2017 International Formulae Group. All rights reserved.

Keywords: Vibrio, Coliforms, physicochemical parameters, aquaculture stations, Ebrié lagoon.

\section{INTRODUCTION}

La lagune Ebrié, avec une superficie de $566 \mathrm{~km}^{2}$, est l'un des écosystèmes aquatiques les plus importants de la Côte d'Ivoire en raison de ses valeurs écologiques et ses exploitations aquacoles. Cependant, de par ses caractéristiques géomorphologiques naturelles, la lagune Ebrié, comme toutes les lagunes, est une étendue d'eau confinée, mal renouvelée, par conséquent vulnérable et dont l'équilibre peut être rapidement modifié sous l'influence de facteurs naturels ou anthropiques (Kouassi, 2005 ; Issola et al., 2008). Elle est le réceptacle des eaux usées domestiques et industrielles et en reçoit environ $40.000 \mathrm{~m}^{3}$ par jour au niveau de la ville d'Abidjan (Koné et al., 2007). Quelques travaux effectués sur la lagune Ebrié ont, en effet, établi des liens directs entre l'état de pollution de la lagune et les déversements d'eaux usées domestiques et industrielles directement déversées dans la lagune sans aucun traitement préalable (Inza et al., 2009). De récentes études ont indiqué un niveau élevé de pollution métallique et organique des eaux et des sédiments de la lagune Ebrié (Tuo et al., 2013). Depuis octobre 1999, des mortalités massives de poissons ont été observées dans deux secteurs de cette lagune, situés dans les départements de Dabou et de Jacqueville. En mai 2013, ce phénomène cyclique s'est accentué, entraînant la fermeture de la pêche et de nombreuses fermes aquacoles dans ces zones lagunaires. Les stations aquacoles de Layo et de
Jacqueville, implantées sur les rives de la lagune Ebrié constituent de véritables foyers de recherche pour le développement de l'élevage du mâchoiron Chrysichthys nigrodigitatus, du silure Heterobranchus longifilis et du tilapia Oreochromis niloticus. A la station aquacole de Layo, l'élevage des poissons est pratiqué dans des étangs en communication avec la lagune via la nappe phréatique. A la station aquacole de Jacqueville, c'est plutôt des enclos lagunaires qui sont utilisés à cet effet. Les produits de pêche issus de ces structures d'élevage pourraient, en cas de contamination par les eaux polluées, poser des problèmes de santé publique et faire l'objet d'une suspension de vente au niveau local et international dans le cadre de la surveillance sanitaire. Ceci pourrait entraîner, par conséquent, une paupérisation des populations locales vivant des activités de pêche et d'aquaculture. En Côte d'Ivoire, la pêche et l'aquaculture occupent une place stratégique, tant sur le plan de la sécurité alimentaire que socioéconomique, il serait plus que nécessaire d'effectuer un meilleur diagnostic des risques de pollution que représentent les eaux des structures d'élevage. La présente étude vise donc à caractériser les paramètres physicochimiques et bactériologiques des eaux des stations aquacoles de Layo et de Jacqueville en vue de maintenir la surveillance de ces eaux. 


\section{MATERIEL ET METHODES}

\section{Site de prélèvement}

Les échantillons d'eau ont été prélevés dans les secteurs IV et V de la lagune Ebrié, au niveau des stations aquacoles (Layo et Jacqueville) et dans le milieu lagunaire environnant (Figure 1). Huit points de prélèvement ont été sélectionnés au niveau de ces sites en fonction des rejets (excréments, eaux usées) et des activités anthropiques. A la station aquacole de Layo (SAL) sous influence des eaux douces de la rivière Agnéby, les étangs E5, E6, E11 et E13 servant au pré-grossissement de différentes espèces de poissons ont été retenus. A environ $1 \mathrm{Km}$ de la SAL, deux points de prélèvement également sous l'influence de la rivière Agnéby ont été retenus dont l'un dans le canal du Niéki, entouré d'une plantation industrielle de bananeraie où se pratique la baignade, la défécation et les activités ménagères; et l'autre dans la lagune à Layo où la pêche est fortement pratiquée. A la station aquacole de Jacqueville (SAJ), des prélèvements ont été effectués dans des enclos de grossissement situés en pleine lagune. Cette station est sous influence fluviale et marine. Au niveau de la lagune à N'djem, un site non loin des installations sanitaires sur pilotis et sous influence fluviale et marine a été retenu. Les coordonnées des différents sites de prélèvement sont marquées dans le Tableau 1.

\section{Echantillonnage}

Six campagnes d'échantillonnage d'eau au niveau de chaque site ont été effectuées de mars à octobre 2015, soit deux campagnes en saison sèche (mars-avril), deux en saison des pluies (juin-juillet) et deux en saison des crues (septembre-octobre). Les échantillons ont été prélevés à environ $20 \mathrm{~cm}$ de profondeur à l'aide de bouteilles borosilicatées de $1000 \mathrm{ml}$ préalablement stérilisées et conservés dans une glacière contenant des carboglaces avant d'être acheminés au laboratoire dans un délai de 4 heures. Au total, 48 échantillons d'eau ont été prélevés à raison de 8 échantillons par point de prélèvement.
Mesures des paramètres physico-chimiques

Le $\mathrm{pH}$, la salinité, la température, la conductivité, les solides totaux dissous (STD) et l'oxygène dissous des eaux ont été mesurés in situ à l'aide d'un multiparamètre YSI 6920 V2-1S (USA). La détermination des matières en suspension (MES) a été faite selon la méthode par centrifugation décrite par Rodier et al. (2009). Le dosage du nitrate $\left(\mathrm{NO}_{3}{ }^{-}\right)$a été effectué par la méthode de réduction au cadmium (HACH, méthode 8039) et celui du nitrite $\left(\mathrm{NO}_{2}^{-}\right)$par la méthode diazotation (HACH, méthode 8507).

\section{Analyses bactériologiques}

La recherche des coliformes fécaux et de Vibrio a été réalisée par la méthode de filtration sur membrane (CEAEQ, 2005). Des volumes de 1,5 et $10 \mathrm{ml}$ de chaque échantillon d'eau ont été filtrés à travers des membranes filtrantes stériles en cellulose, de porosité 0,45 $\mu \mathrm{m}$ (Sartorius Stedim Biotech, Allemagne). Les membranes ont été ensuite placées dans des boîtes de Pétri sur les géloses Eosine Bleu de Méthylène (EMB) (BIO-RAD, France) pour les coliformes fécaux et Thiosulfate Citrate Bile Saccharose (TCBS) (HIMEDIA, Inde) pour les Vibrio. Les boîtes de Pétri ont été incubées à $44^{\circ} \mathrm{C}$ pendant 24 heures pour les coliformes fécaux et à $37^{\circ} \mathrm{C}$ pendant 24 heures pour les Vibrio. Après incubation, les colonies violettes à brunes ayant ou pas un reflet métallique sur EMB ont été retenues pour le dénombrement des coliformes fécaux, tandis que les colonies jaunes et vertes sur TCBS ont été retenues pour les Vibrio. Le nombre de colonies a été exprimé sous forme d'Unités Formant Colonies $/ 100 \mathrm{ml}$ d'eau, puis transformé en logarithme.

\section{Analyses statistiques}

L'analyse statistique a été réalisée à l'aide du logiciel ADE4. A partir de l'analyse en composantes principales (ACP), la réalisation de la carte factorielle, la corrélation entre les différents paramètres et la classification des points de prélèvement en fonction des paramètres physico-chimiques ont été établies au seuil de signification $\alpha=$ 0,05 . 


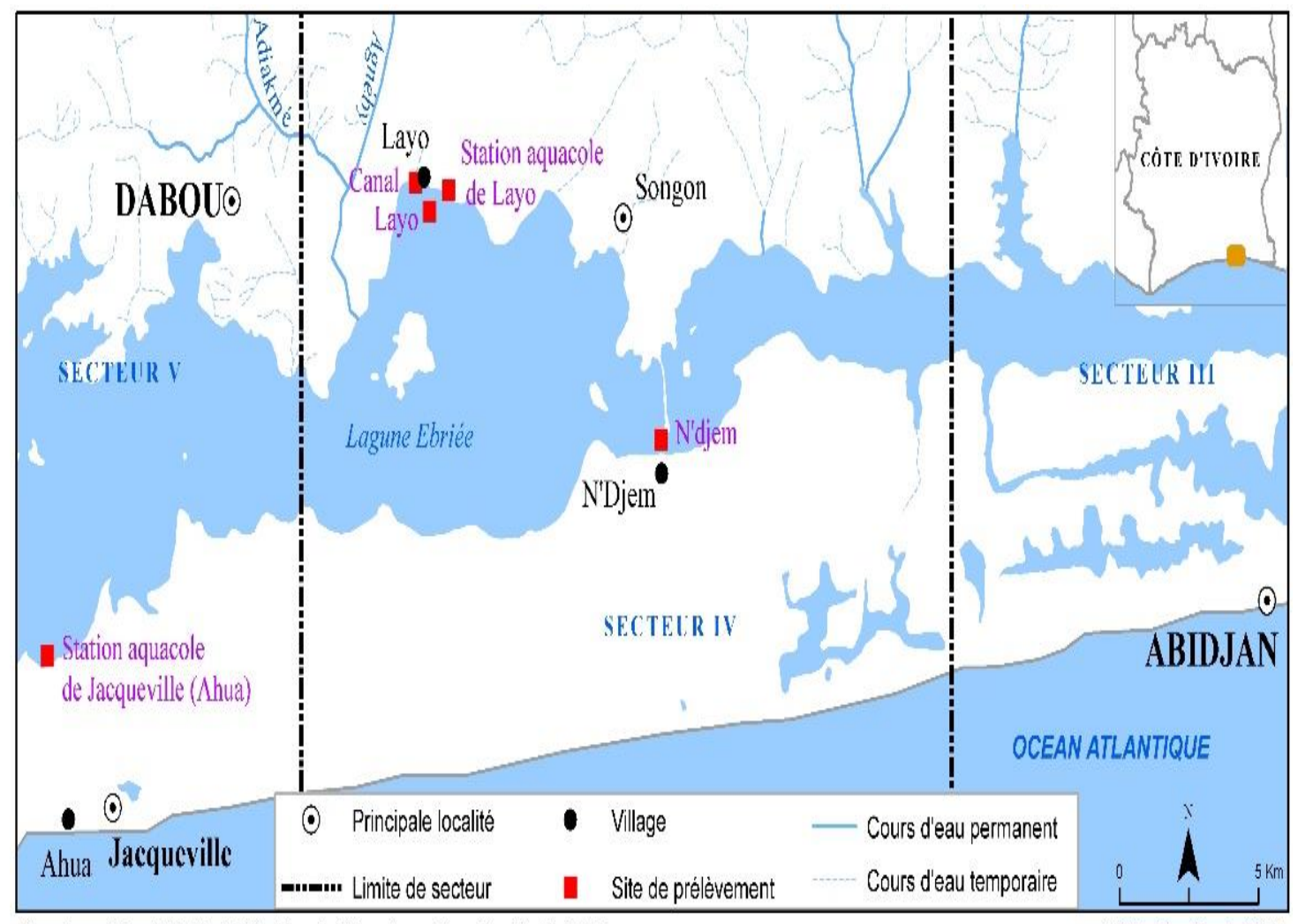

Donnée spatiale: CNTIG, 2012 - Donnée thématique: Enquêtes Toulè, 2017

Réalisation: Toulé, 2017

Figure 1 : Localisation de la zone d'étude et des points d'échantillonnage.

Tableau 1 : Coordonnées géographiques des différents sites de prélèvement.

\begin{tabular}{|c|c|c|c|c|}
\hline SAL & Canal & Layo & SAJ & N'djem \\
\hline $\mathrm{N} \mathrm{05^{ \circ } 1 9 ^ { \prime } 3 0 , 6 ^ { \prime \prime }}$ & N 05¹9'34,5" & N 0519'273" & $\mathrm{N} 05^{\circ} 13^{\prime} 50,9^{\prime \prime}$ & 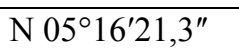 \\
\hline $\mathrm{W} 004^{\circ} 18^{\prime} 45,5^{\prime \prime}$ & W $004^{\circ} 19^{\prime} 04,2^{\prime \prime}$ & W $004^{\circ} 19^{\prime} 079^{\prime \prime}$ & $\mathrm{W} 004^{\circ} 26^{\prime} 25,1^{\prime \prime}$ & W $004^{\circ} 14^{\prime} 23,4^{\prime \prime}$ \\
\hline
\end{tabular}

SAL : station aquacole de Layo, SAJ : station aquacole de Jacqueville. 


\section{RESULTATS}

Les variations spatio-temporelles des paramètres physico-chimiques des eaux sont présentées dans le Tableau 2. Les valeurs moyennes de la température des eaux des différents points de prélèvement varient de 28,2 à $30,1^{\circ} \mathrm{C}$, celles du $\mathrm{pH}$ de 5,7 à 7,6 et de 4,3 à $57,3 \mathrm{mg} / \mathrm{l}$ pour les teneurs en MES. Les valeurs les plus élevées de la température, du $\mathrm{pH}$ et de la teneur en MES ont été enregistrées au niveau de l'étang E6, avec pour valeurs respectives $30,1{ }^{\circ} \mathrm{C}, 7,6$ et $57,3 \mathrm{mg} / \mathrm{l}$. Les valeurs les plus faibles ont été enregistrées dans le canal pour le $\mathrm{pH}(5,7)$, dans le milieu lagunaire à Layo pour la température $\left(28,2{ }^{\circ} \mathrm{C}\right)$ et à la SAJ pour les teneurs en MES $(4,3 \mathrm{mg} / \mathrm{l})$. Les teneurs en STD étaient de l'ordre de 0,18 à $10,21 \mathrm{mg} / \mathrm{l}$ et celles en oxygène dissous ont varié de 2,43 à 7,96 $\mathrm{mg} / \mathrm{l}$. La salinité et la conductivité ont respectivement varié de 0,1 à $6,52 \%$ et de 0,23 à $5,84 \mathrm{mS} / \mathrm{cm}$. Les valeurs les plus élevées pour la salinité et la conductivité ont été observées à $\mathrm{N}^{\prime}$ djem $(6,52 \%$ et $5,84 \mathrm{mS} / \mathrm{cm})$ et les plus faibles dans le canal $(0,1 \%$ et 0,23 $\mathrm{mS} / \mathrm{cm}$ ). Les concentrations en $\mathrm{NO} 3$ - et en $\mathrm{NO} 2-$ étaient faibles et ont varié respectivement de 0,6 à $1,75 \mathrm{mg} / \mathrm{l}$ et de 0,002 à $0,038 \mathrm{mg} / \mathrm{l}$. Les variations saisonnières, plus ou moins faibles sont caractérisées par des valeurs maximales de $\mathrm{pH}$, de salinité, de température, de conductivité électrique, des teneurs en STD et en oxygène dissous pendant la saison sèche contrairement aux teneurs de NO3-, de NO2- et de MES dont les valeurs les plus élevées ont été obtenues en saison des crues.

Les variations spatio-temporelles des charges bactériologiques des eaux sont présentées dans le Tableau 3. Les coliformes fécaux (CF) et les Vibrio sont présents au niveau de tous les points de prélèvement et leurs charges moyennes ont varié respectivement de 3,21 à 4,29 $\log \mathrm{UFC} / 100 \mathrm{ml}$ et 3,3 à 4,26 log UFC/100 ml. Les charges les plus élevées ont été enregistrées à N'djem pour les coliformes fécaux $(4,29 \log \mathrm{UFC} / 100 \mathrm{ml})$ et au niveau de l'étang E5 pour les Vibrio $(4,26$ $\log \mathrm{UFC} / 100 \mathrm{ml})$. Les charges minimales ont été observées à la $\mathrm{SAJ}$ pour les coliformes fécaux $(3,21 \log \mathrm{UFC} / 100 \mathrm{ml})$ et dans la lagune à Layo pour les Vibrio $(3,3 \log \mathrm{UFC} / 100 \mathrm{ml})$. Les variations saisonnières des charges bactériologiques sont faibles.
Une analyse en composantes principales normées (ACPN) a été réalisée dans le but de mettre en évidence les relations pouvant exister entre les différents paramètres au niveau des points de prélèvement (Figure 2). Le plan factoriel résumait $70,77 \%$ de l'information globale. L'axe F1, avec $43,85 \%$ de la variance totale est représentée par la conductivité, la salinité, les STD, les MES, la température, l'oxygène dissous et le NO2-. Les regroupements de ces paramètres dans les pôles opposés de cet axe montrent que la composition de l'eau est influencée par deux phénomènes différents. Les paramètres salinité, conductivité et STD renseignent sur le degré de la pollution minérale des eaux. Il se crée un gradient de minéralisation croissant allant du pôle positif vers le pôle négatif de l'axe. Les paramètres température et MES déterminent le degré de pollution organique des eaux. Un gradient de pollution organique croissant se crée allant du pôle négatif vers le pôle positif. L'essentiel de l'information apportée par l'axe F2 (26,92\% de variance) est expliqué par les paramètres bactériologiques (coliformes fécaux et Vibrio), le pH et le NO3-. Les observations correspondant aux étangs E5, E6, E11 et E13 se projettent à droite du plan factoriel (B) dont les eaux sont caractérisées par une faible minéralisation et une forte anthropisation. Par contre, les observations correspondant au canal, à Layo, à la SAJ et à N'djem se projettent à gauche du plan factoriel (A), avec des eaux caractérisées par une faible minéralisation et anthropisation pour le canal et des eaux caractérisées par une forte minéralisation et une faible anthropisation pour Layo, la SAJ et N'djem (Figure 2).

Le Tableau 4 présente la matrice de corrélation des paramètres étudiés. Des corrélations significativement positives ont été observées entre le $\mathrm{pH}$, la température, les MES, l'oxygène dissous et les NO3-. Des corrélations significativement positives ont également été observées entre la salinité, la conductivité, les STD et les NO2-. Une corrélation négative significative a été observée entre les MES et le groupe constitué de la conductivité et des NO2- Une corrélation positive significative a été observée entre les coliformes fécaux et les Vibrio. Aucune corrélation n'a été observée entre les paramètres physico-chimiques et les paramètres bactériologiques. 
Tableau 2 : Variation spatio-temporelle des paramètres physico-chimiques des eaux.

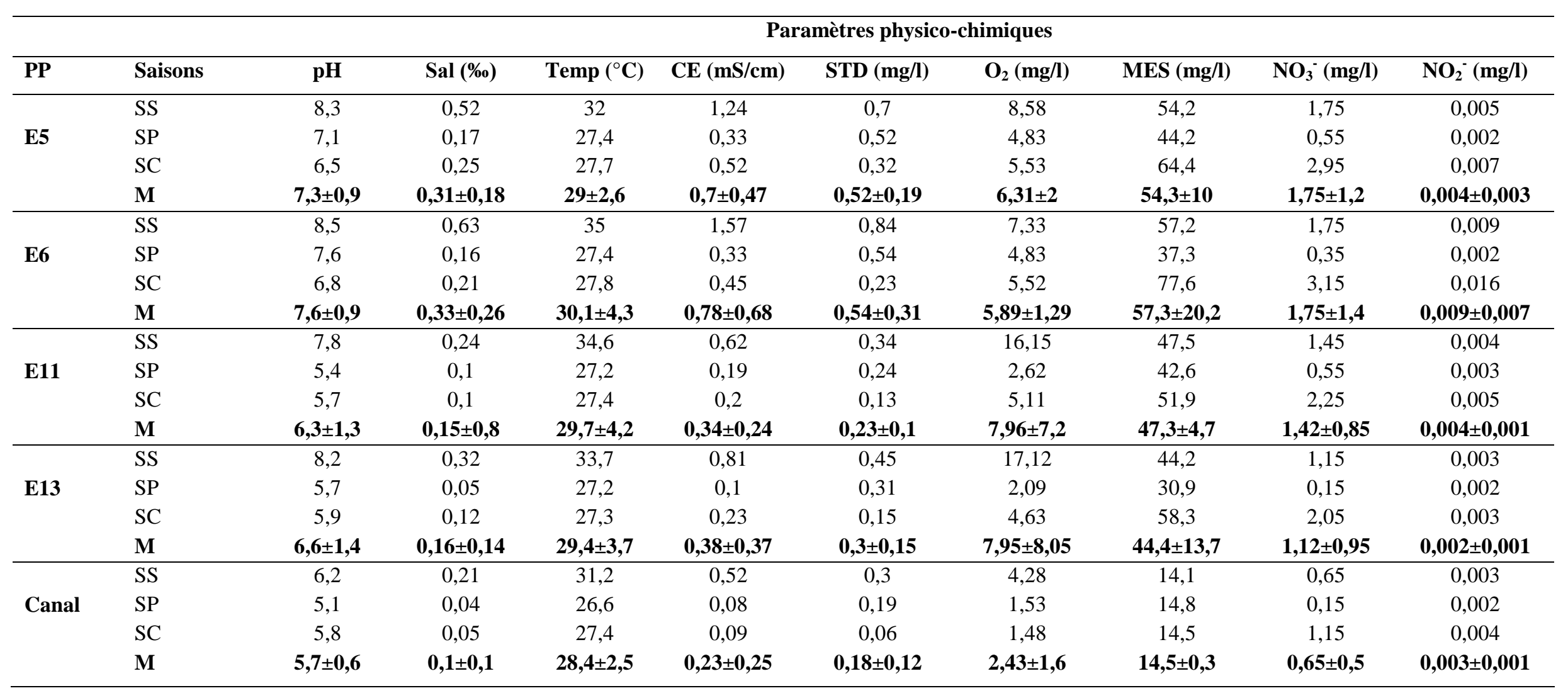


A. C. TOULE et al. / Int. J. Biol. Chem. Sci. 11(6): 2842-2855, 2017

\begin{tabular}{|c|c|c|c|c|c|c|c|c|c|c|}
\hline & SS & 6,1 & 3,42 & 31,4 & 7,2 & 4,13 & 5,05 & 21,1 & 0,65 & 0,003 \\
\hline \multirow[t]{4}{*}{ Layo } & SP & 5,5 & 0,04 & 26,4 & 0,09 & 2,69 & 0,78 & 23,3 & 0,15 & 0,002 \\
\hline & $\mathrm{SC}$ & 5,9 & 0,97 & 26,9 & 1,96 & 1,23 & 3,51 & 19,3 & 1 & 0,004 \\
\hline & $\mathbf{M}$ & $5,8 \pm 0,3$ & $1,48 \pm 1,75$ & $28,2 \pm 2,8$ & $3,09 \pm 3,69$ & $2,69 \pm 1,45$ & $3,11 \pm 2,17$ & $21,2 \pm 2$ & $0,6 \pm 0,43$ & $0,003 \pm 0,001$ \\
\hline & SS & 7,4 & 3,71 & 30,5 & 7,68 & 4,44 & 8,81 & 4,2 & 1,05 & 0,028 \\
\hline \multirow[t]{4}{*}{ SAJ } & SP & 6,4 & 2 & 27,3 & 5,12 & 3,46 & 6,21 & 5,8 & 0,65 & 0,043 \\
\hline & $\mathrm{SC}$ & 6,6 & 2,56 & 27,6 & 3,98 & 2,47 & 6,41 & 3 & 1,45 & 0,045 \\
\hline & $\mathbf{M}$ & $6,8 \pm 0,5$ & $2,76 \pm 0,87$ & $28,5 \pm 1,8$ & $5,59 \pm 1,89$ & $3,45 \pm 0,98$ & $7,14 \pm 1,45$ & $4,3 \pm 1,4$ & $1,05 \pm 0,4$ & $0,038 \pm 0,009$ \\
\hline & SS & 7,3 & 10,42 & 31,1 & 12,78 & 11,57 & 4,56 & 24 & 1,45 & 0,027 \\
\hline \multirow[t]{3}{*}{ N'djem } & SP & 6,5 & 1,29 & 27,5 & 2,57 & 10,22 & 3,75 & 28,7 & 0,85 & 0,02 \\
\hline & $\mathrm{SC}$ & 6,3 & 7,84 & 27,6 & 2,15 & 8,86 & 4,17 & 19,7 & 1,95 & 0,033 \\
\hline & $\mathbf{M}$ & $6,7 \pm 0,5$ & $6,52 \pm 4,71$ & $28,7 \pm 2,1$ & $5,84 \pm 6,02$ & $10,21 \pm 1,35$ & $4,16 \pm 0,41$ & $24,1 \pm 4,5$ & $1,42 \pm 0,55$ & $0,026 \pm 0,007$ \\
\hline
\end{tabular}

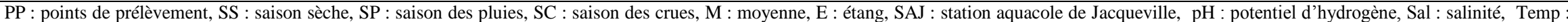
température, $\mathrm{CE}$ : conductivité électrique, $\mathrm{STD}$ : solides totaux dissous, $\mathrm{O}_{2}$ : oxygène dissous, MES : matières en suspension. 
Tableau 3 : Variation spatio-temporelle des charges bactériologiques des eaux.

\begin{tabular}{|c|c|c|c|}
\hline \multirow{2}{*}{ PP } & \multirow[b]{2}{*}{ Saisons } & \multicolumn{2}{|c|}{ Charges bactériologiques $(\log \mathrm{UFC} / 100 \mathrm{ml})$} \\
\hline & & Coliformes fécaux (CF) & Vibrio \\
\hline \multirow{4}{*}{ E5 } & SS & 3,85 & 4,57 \\
\hline & SP & 3,72 & 4,06 \\
\hline & SS & 3,75 & 4,15 \\
\hline & $\mathbf{M}$ & $\mathbf{3 , 7 7 \pm 0 , 0 7}$ & $4,26 \pm 0,27$ \\
\hline \multirow{4}{*}{ E6 } & SS & 3,73 & 4,04 \\
\hline & SP & 3,65 & 3,29 \\
\hline & $\mathrm{SC}$ & 3,68 & 3,35 \\
\hline & $\mathbf{M}$ & $3,67 \pm 0,02$ & $3,56 \pm 0,42$ \\
\hline \multirow{4}{*}{ E11 } & SS & 3,76 & 4,34 \\
\hline & SP & 3,73 & 3,19 \\
\hline & $\mathrm{SC}$ & 3,56 & 3,3 \\
\hline & $\mathbf{M}$ & $3,75 \pm 0,02$ & $3,61 \pm 0,63$ \\
\hline \multirow{4}{*}{ E13 } & SS & 3,75 & 3,86 \\
\hline & SP & 3,72 & 3,12 \\
\hline & $\mathrm{SC}$ & 3,57 & 3,5 \\
\hline & $\mathbf{M}$ & $3,65 \pm 0,11$ & $3,49 \pm 0,53$ \\
\hline \multirow{4}{*}{ Canal } & SS & 4,05 & 4,35 \\
\hline & SP & 3,88 & 3,38 \\
\hline & $\mathrm{SC}$ & 3,84 & 3,42 \\
\hline & $\mathbf{M}$ & $3,92 \pm 0,11$ & $3,82 \pm 0,48$ \\
\hline \multirow{4}{*}{ Layo } & SS & 3,63 & 3,56 \\
\hline & SP & 3,72 & 3,18 \\
\hline & $\mathrm{SC}$ & 3,55 & 3,17 \\
\hline & $\mathbf{M}$ & $3,63 \pm 0,08$ & $3,3 \pm 0,22$ \\
\hline \multirow[t]{4}{*}{ SAJ } & SS & 2,86 & 3,71 \\
\hline & SP & 3,41 & 3,67 \\
\hline & $\mathrm{SC}$ & 3,35 & 3,45 \\
\hline & $\mathbf{M}$ & $3,21 \pm 0,3$ & $3,61 \pm 0,14$ \\
\hline \multirow{4}{*}{ N'djem } & $\mathrm{SS}$ & 4,16 & 4,41 \\
\hline & SP & 4,38 & 4,13 \\
\hline & $\mathrm{SC}$ & 4,32 & 3,98 \\
\hline & $\mathbf{M}$ & $4,29 \pm 0,11$ & $4,17 \pm 0,22$ \\
\hline
\end{tabular}

PP : point de prélèvement, SS : saison sèche, SP : saison des pluies, $\mathrm{SC}$ : saison des crues, M : moyenne, E : étang, AJ : station aquacole de Jacqueville.

Tableau 4 : Matrice de corrélation de Bravais Pearson entre les variables étudiées.

\begin{tabular}{|c|c|c|c|c|c|c|c|c|c|c|c|}
\hline & $\mathrm{pH}$ & Sal & Temp & $\mathrm{CE}$ & STD & $\mathrm{O}_{2}$ & MES & $\mathrm{NO}_{3}{ }^{-}$ & $\mathrm{NO}_{2}^{-}$ & $\mathrm{CF}$ & Vibrio \\
\hline pH & 1 & & & & & & & & & & \\
\hline Sal & $-0,19$ & 1 & & & & & & & & & \\
\hline Temp & 0,65 & $-0,38$ & 1 & & & & & & & & \\
\hline CE & $-0,16$ & 0,89 & $-0,53$ & 1 & & & & & & & \\
\hline STD & $-0,22$ & 0,99 & $-0,37$ & 0,86 & 1 & & & & & & \\
\hline $\mathrm{O}_{2}$ & 0,55 & $-0,25$ & 0,63 & $-0,17$ & $-0,29$ & 1 & & & & & \\
\hline MES & 0,65 & $-0,46$ & 0,85 & $-0,65$ & $-0,42$ & 0,47 & 1 & & & & \\
\hline $\mathrm{NO}_{3}^{-}$ & 0,84 & 0,03 & 0,74 & $-0,14$ & 0,02 & 0,53 & 0,76 & 1 & & & \\
\hline $\mathrm{NO}_{2}^{-}$ & 0,11 & 0,73 & $-0,30$ & 0,87 & 0,66 & 0,12 & $-0,57$ & 0,07 & 1 & & \\
\hline CF & $-0,29$ & 0,42 & 0,01 & $-0,01$ & 0,48 & $-0,46$ & 0,15 & 0,16 & $-0,21$ & 1 & \\
\hline Vibrio & 0,19 & 0,39 & $-0,08$ & 0,14 & 0,39 & $-0,11$ & 0,12 & 0,49 & 0,19 & 0,59 & 1 \\
\hline
\end{tabular}

En gras : valeurs de $\mathrm{r}$ significatives pour $\mathrm{P}<0,05$. Sal : salinité, Temp : température, CE : conductivité électrique, STD : solides totaux dissous, $\mathrm{O}_{2}$ : oxygène dissous, MES : matières en suspension, $\mathrm{NO}_{3}{ }^{-}$: nitrate, $\mathrm{NO}_{2}{ }^{-}$: nitrite, $\mathrm{CF}$ : coliformes totaux. 


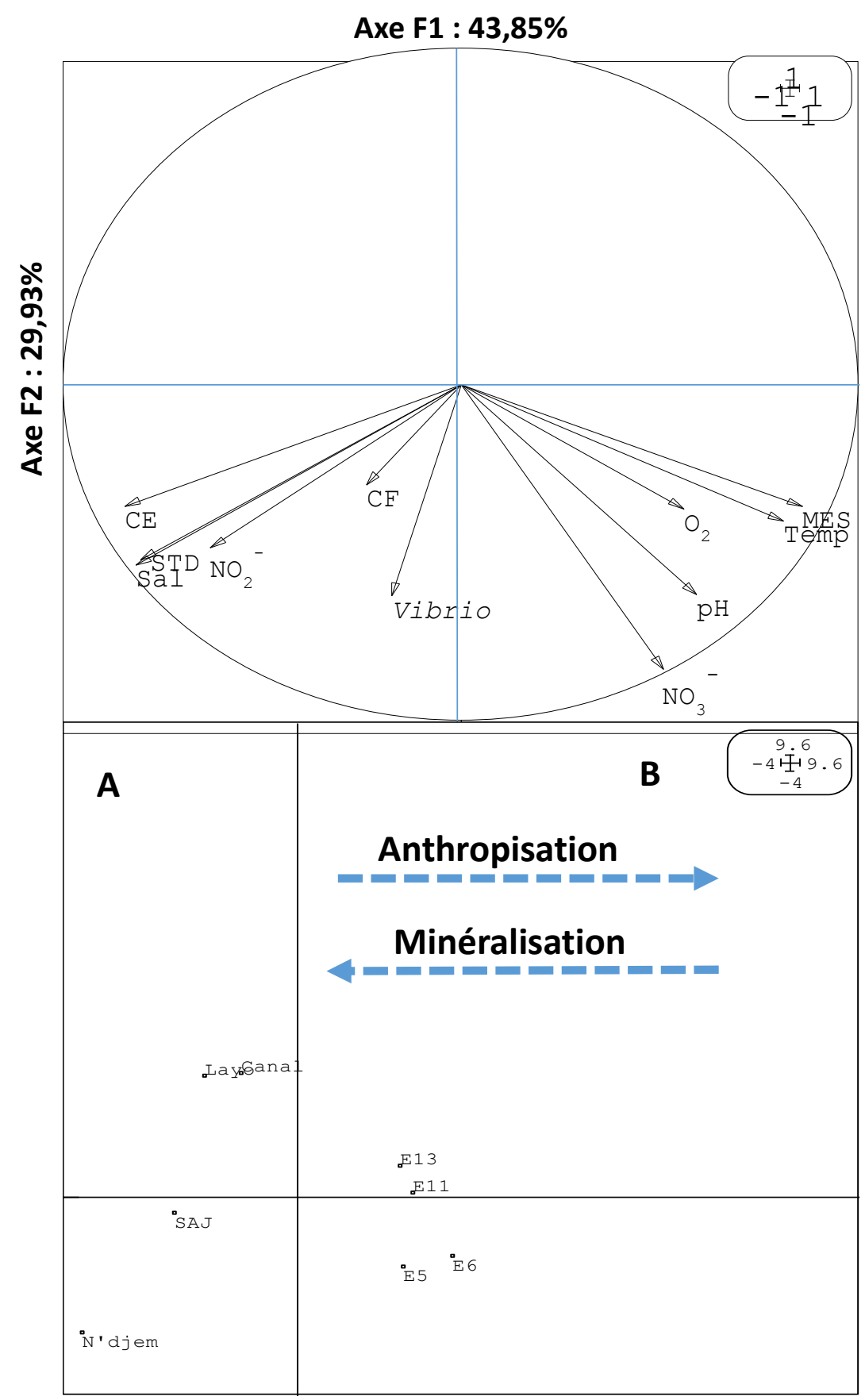

Figure 2 : Plan 1-2 de l'analyse en composantes principales normées (ACPN). Haut : projection des variables, bas : représentation des observations des points de prélèvement. Sal : Salinité, Temp : Température, CE : Conductivité électrique, STD : Solides totaux dissous, O2 : Oxygène dissous, MES : Matières en suspension. SAJ : Station aquacole de Jacqueville, E : Etang. 


\section{DISCUSSION}

Le suivi des paramètres physicochimiques et bactériologiques a montré des variations spatio-temporelles pour chaque paramètre sur la période d'étude. La variation de la température $\left(28,2\right.$ à $\left.30,1^{\circ} \mathrm{C}\right)$ des eaux au niveau des stations aquacoles et du milieu lagunaire s'expliquerait par le fait que la Côte d'Ivoire a un climat tropical (Inza et al., 2009). La température des eaux était élevée $\left(30,5-35{ }^{\circ} \mathrm{C}\right)$ en saison sèche et faible $(26,4-$ $27,4{ }^{\circ} \mathrm{C}$ ) en saison des pluies. Selon Lwamba et al. (2015), la variation de la température des eaux de surface est étroitement liée au rayonnement solaire. Les teneurs en MES obtenues au cours du présent travail étaient supérieures à celles de la lagune Aby (6,1 $\mathrm{mg} / \mathrm{l}$ à $15 \mathrm{mg} / \mathrm{l}$ ) rapportées par Kambiré et al. (2012) et à celles de la lagune de Fresco $(25,06 \mathrm{mg} / \mathrm{l}$ à $47,69 \mathrm{mg} / \mathrm{l})$ rapportées par Issola et al. (2008). Cette différence pourrait être liée à l'hydrodynamisme, à la géomorphologie ou à la pression anthropique autour de ces écosystèmes. Les étangs de la station aquacole de Layo ont enregistré de fortes températures et teneurs en MES par rapport aux autres points de prélèvement (canal, station aquacole de Jacqueville, milieu lagunaire à Layo et à N'djem) par le fait qu'ils sont des écosystèmes fermés où le renouvellement des eaux est rare. Dans les structures d'élevage, les poissons sont nourris à l'aliment artificiel. De ce fait, les MES pourraient être attribués aux énormes quantités de matières organiques produites à partir des aliments non consommés et des déchets des métabolites des poissons. La remise en suspension des MES lors des activités aquacoles (sexage, pêche, tri, transfert, etc.), la pénétration des pluies et le transport par ruissellement de particules de terre et de végétal vers les étangs et les enclos pourraient occasionner une augmentation des teneurs en MES. Dans le canal et le milieu lagunaire à Layo et à N'djem, les MES seraient attribuées aux eaux continentales provenant de la rivière Agnéby qui, après avoir parcouru son bassin versant y déverse ses effluents. De plus, ces milieux sont des zones d'intenses activités anthropiques notamment, la baignade, la défécation, la lessive, la pêche, la navigation.

Les eaux dans les étangs ont également présenté des valeurs variables de $\mathrm{pH}$, avec des $\mathrm{pH}$ alcalins $(7,8-8,5)$ pendant la saison sèche et des pH par moment acides $(5,4-5,7)$ ou basiques $(7,6)$ pendant les saisons de pluies et des crues. Cette variation du $\mathrm{pH}$ s'expliquerait par le caractère mixte de l'alimentation-nappe phréatique et précipitations, par le phénomène de l'évaporation et par les fortes activités biologiques, en particulier celles attribuables aux communautés phytoplanctoniques comme indiquée par Adingra et al. (2007). Dans le canal et la lagune à Layo, les eaux étaient en permanence acides en raison de leur influence par la rivière Agnéby. Les eaux de la station aquacole de Jacqueville et de N'djem ont présenté des $\mathrm{pH}$ acides $(6,4-6,5)$ pendant les saisons des pluies et des crues et des $\mathrm{pH}$ légèrement alcalins $(7,3-7,4)$ pendant la saison sèche. Cette légère alcalinité pendant la saison sèche laisse transparaître l'influence des eaux marines qui sont généralement basiques par rapport à celles d'origine continentale. Selon Inza et al. (2009), les valeurs des pH les plus élevées s'observent pendant la période d'étiage (forte influence océanique) et les plus faibles pendant la période de pluies et de crues.

Les variations spatio-temporelles de la salinité et de la conductivité ont présenté une évolution parallèle. En effet, les valeurs de ces deux paramètres ont augmenté pour atteindre leur maximum pendant la saison sèche et diminuer en saisons des pluies et des crues. De tels résultats indiquent une étroite corrélation entre ces deux paramètres comme l'ont déjà souligné Kambiré et al. (2014). L'augmentation de la salinité et de la conductivité en saison sèche pourrait s'expliquer par une importante évaporation de l'eau due à l'élévation de la température et à une entrée massive d'eau océanique dans la lagune comme l'a rapporté Traoré et al. (2012). Dans cette étude, les valeurs moyennes de la salinité $(0,1-6,52 \%)$ étaient proches de celles $(0,4-6,9 \%)$ mesurées par 
Inza et al. (2009). Cependant, les valeurs de la conductivité $\quad(0,23-5,84 \mathrm{mS} / \mathrm{cm})$ étaient inférieures à celles $(0,31-9 \mathrm{mS} / \mathrm{cm})$ mesurées par Affian et al. (2002) dans la même lagune Ebrié et supérieures à celles (0,06-2,13 $\mathrm{mS} / \mathrm{cm}$ ) mesurées par Kambiré et al. (2012) dans la lagune Aby. La station aquacole de Jacqueville et $\mathrm{N}$ 'djem, plus proches de la zone océanique, ont présenté les valeurs les plus élevées de salinité et de conductivité par rapport à la station aquacole de Layo, au canal et à Layo qui sont plus sous l'influence des eaux continentales. L'évolution spatiotemporelle des solides totaux dissous était identique à celle de la salinité et de la conductivité.

Les concentrations en oxygène dissous étaient plus importantes en saison sèche qu'en saisons des pluies et des crues au niveau de tous les points prélèvement. Cela s'expliquerait par le fait qu'en saisons de pluies et de crues, les eaux dans les secteurs $\mathrm{IV}$ et $\mathrm{V}$ de la lagune Ebrié soumises aux apports continentaux s'appauvrissent en oxygène comme indiqué par Djadji (2015). En effet, les eaux des rivières forestières supportant de grandes plantations industrielles et d'une forte turbidité sont chargées d'énormes quantités de matières organiques et d'acides humiques et elles ne permettent pas à la photosynthèse de compenser les pertes dues à la respiration des organismes et l'oxydation des matières organiques détritiques qu'elles charrient (Djadji, 2015).

Les teneurs des $\mathrm{NO}_{3}^{-}(0,06$ à $1,75 \mathrm{mg} / \mathrm{l})$ et celles des $\mathrm{NO}_{2}^{-}(0,002$ à $0,038 \mathrm{mg} / \mathrm{l})$ dans les eaux étaient relativement faibles par rapport aux normes OMS (2004) qui sont de $50 \mathrm{mg} / \mathrm{l}$ et $0,1 \mathrm{mg} / \mathrm{l}$ respectivement. Les quantités de $\mathrm{NO}_{3}{ }^{-}$et de $\mathrm{NO}_{2}{ }^{-}$relevées dans les étangs de la station aquacole de Layo ont probablement pour origine une forte minéralisation de l'azote organique pendant le processus d'assèchement et de chaulage, les apports en composés minéraux par les eaux de la nappe phréatique, les aliments artificiels non consommés et les déchets métaboliques des poissons en élevage. Dans les enclos de la station aquacole de Jacqueville, en plus des apports en éléments minéraux par les aliments non consommés et les déchets métaboliques des poissons en élevage, les teneurs en sels nutritifs seraient induites par les apports d'eaux marines riches en minéraux comme à N'djem. Au canal et à Layo, les teneurs en sels nutritifs relevées sont probablement dues aux apports des pollutions par la rivière Agnéby, dont le bassin versant supporte plusieurs plantations industrielles.

De fortes charges de coliformes fécaux et de Vibrio ont été mises en évidence dans les eaux des structures d'élevage et celles de la lagune. Dans les étangs de la station aquacole de Layo, les charges de coliformes fécaux et de Vibrio étaient élevées que celles dans les enclos de la station aquacole de Jacqueville. $\mathrm{La}$ différence du degré de pollution bactériologique entre ces deux stations était liée à la géomorphologie et à l'hydrodynamisme de ces deux structures. En effet, les étangs de la station aquacole de Layo sont des milieux fermés où la circulation horizontale et les échanges verticaux sont ralentis. Dans ces bassins d'élevage, les apports alimentaires et les rejets des métabolites des poissons en captivité s'accumulent dans les sédiments, offrant ainsi une bonne source de nutriments aux différentes bactéries qui s'y trouvent, avec pour conséquence une pollution aigue. Selon Hartel et al. (2005), les sédiments peuvent servir de réservoir pour les bactéries fécales durant le cycle hydroclimatique. De nombreux auteurs font état de la capacité des sédiments à prolonger la survie des microorganismes fécaux (Whitman et Nevers, 2003 ; Ishii et al., 2006 ; Kon et al., 2007). Les enclos de la station aquacole de Jacqueville, par contre, sont des milieux ouverts où l'hydrodynamisme est actif, avec un renouvellement et une oxygénation des eaux, ce qui a pour conséquence une faible pollution. Selon Adingra et al. (2007), l'accroissement du nombre des Vibrio dans les eaux des bassins d'élevage pourrait être en relation avec la nourriture employée. La présence de ces germes en structure aquacole pourrait constituer un risque important, 
d'abord pour les poissons en élevage et ensuite, pour les consommateurs. En effet, les vibrions principalement $V$. parahaemolyticus, $V$. alginolyticus et $V$. anguillarum sont responsables d'infections touchant des spécimens variés de poissons (Ben KahlaNakbi et al., 2006). Selon Cohen et al. (2007), certaines espèces bactériennes du genre Vibrio considérées aujourd'hui comme des pathogènes émergents sont impliquées dans les infections d'origine alimentaire chez l'homme après ingestion de produits de pêche contaminés, posant ainsi un problème de santé publique. De plus, ces milieux contaminés par Vibrio pourraient constituer un danger pour les aquaculteurs lors d'un contact direct avec les eaux, causant ainsi des dermatoses ou septicémies. Dans le canal et la lagune à Layo et à N'djem, les fortes densités de coliformes fécaux et de Vibrio relevées seraient dues au fort degré d'anthropisation causé par la défécation anarchique, les rejets d'ordures et d'eaux usées ménagères par les populations riveraines dans la lagune. De plus, au niveau de Layo et du canal, il convient d'indiquer que la rivière Agnéby y déverse ses effluents après avoir parcouru son bassin versant. Ces effluents sont susceptibles de contenir des microorganismes qui pourraient être responsables de la contamination fécale. Selon Elmanama et al. (2006), l'augmentation de la densité des indicateurs fécaux en zone côtière a été souvent sous influence des rejets d'eaux usées urbaines et agricoles, véhiculés en amont par les eaux de ruissellement drainant les rejets de réseaux sanitaires et des bassins versants. De plus, la richesse en matière organique des milieux ainsi qu'un attachement aux surfaces planctoniques contenant de la chitine favorisent la survie et la multiplication des espèces de Vibrio (Canesi et al., 2006 ; Vezzulli et al., 2008).

Une corrélation positive entre les coliformes fécaux et les Vibrio a été observée, ce qui traduit une probabilité de détection simultanée de ces deux groupes bactériens lors d'une étude bactériologique des eaux de surface. Aucune corrélation entre les paramètres bactériologiques et les paramètres physico-chimiques n'a été observée. Aucune variation saisonnière bien marquée n'a également été observée pour les coliformes fécaux et les Vibrio dans les eaux de surface. $\mathrm{La}$ pollution bactérienne des stations d'élevage serait probablement dominée par une source ponctuelle ou sous l'influence des activités aquacoles (Alimentation, curage, pêche...), des rejets (métabolites des poissons, déchets domestiques) et des processus hydrodynamiques à savoir, la dilution, la dispersion, la sédimentation et la remise en suspension dans le milieu aquatique.

\section{Conclusion}

Les résultats de ce travail ont permis de disposer de données sur les paramètres physico-chimiques et bactériologiques des eaux des stations de Layo et de Jacqueville, ainsi que celles du milieu lagunaire environnant. Les concentrations des sels nutritifs (nitrate, nitrite) sont dans les limites autorisées selon l'OMS. La station aquacole de Layo a présenté de fortes teneurs en matières en suspension par rapport à la station aquacole de Jacqueville. Les eaux de la station aquacole de Layo, sous influence continentale, ont présenté les valeurs de salinité et de conductivité les plus faibles par rapport à la station aquacole de Jacqueville. Les eaux de ces deux structures d'élevage et celles du milieu lagunaire hébergeaient de fortes densités de bactéries fécales et de vibrions du fait des activités aquacoles, des rejets de déchets des poissons en captivité et des déchets domestiques. De ce fait, il serait plus que nécessaire de renouveler constamment les eaux des étangs afin d'éviter une forte contamination et des mortalités massives des poissons. Aussi, une éducation sanitaire à l'endroit des populations riveraines de la lagune Ebrié serait nécessaire pour prévenir les risques liés à certaines mauvaises pratiques d'hygiène afin d'éviter des épidémies éventuelles.

\section{CONFLITS D'INTERETS}

Les auteurs déclarent qu'il n'y a aucun conflit d'intérêts. 


\section{CONTRIBUTIONS DES AUTEURS}

NK-N' et ACT ont contribué aux campagnes d'échantillonnages, aux analyses de laboratoires et à la rédaction de l'article ; OK, RK-N et MK ont contribué à la lecture et la correction du manuscrit; Supervision de toutes les opérations a été assurée par AAA.

\section{REMERCIEMENTS}

Nous remercions le Centre Ivoirien Anti-pollution (CIAPOL) qui a mis à notre disposition les appareils et équipements qui ont permis les mesures des paramètres physico-chimiques.

\section{REFERENCES}

Adingra AA. 2007. Pollutions organique et bactérienne des eaux de Côte d'Ivoire: cas d'une zone rurale (station aquacole expérimentale de Layo) et d'une zone urbaine lagune Ebrié. Thèse de Doctorat, Université de Cocody, Abidjan (Côte d'Ivoire), p. 184.

Affian K, Djagoua EV, Kouame KF, Gioan P, Biemi J. 2002. Etude par télédétection aéroportée d'un environnement lagunaire tropicale : cas de la lagune Ebrié en Côte d'Ivoire. Télédétection, 2(4): 233-242.

Ben Kahla-Nakbi A, Lachkar-Kacem H, Elmnasser N, Jihène Cheriaa J, Bakhrouf A. 2006. Aspects histopathologiques associés à l'infection du loup et de la daurade par Vibrio alginolyticus. Mar. Life, 16: 3136.

Canesi L, Zampini M, Repetto B, Pezzati E, Schito MA, Vezzulli L, Gallo G, Pruzzo C. 2006. Vibrio interactions with biotic and abiotic surfaces in seawater and links with pathogenicity. In: Marine Sciences and Public Health, CIESM workshop Monographs (31), Geneve 27-30 September: 73-76.

CEAEQ (Centre d'Expertise en Analyse Environnementale du Québec). 2005. Recherche et dénombrement des coliformes fécaux (thermotolérants) et confirmation à l'espèce Escherichia coli : méthode par filtration sur membrane. MA. 700-Fec. Ec 1.0, Rév. 2, Ministère du Développement durable, de l'Environnement et des Parcs du Québec, p. 20.

Cohen N, Karib H, Ait Saïd J, Lemee L, Guenole A, Quilici ML. 2007. Prévalence des vibrions potentiellement pathogènes dans les produits de la pêche commercialisés à Casablanca (Maroc). Rev. Méd. Vét., 158: $562-568$.

Djadji ELG. 2015. Etude des traits biologiques et du régime alimentaire de deux espèces de Mugilidae, Liza falcipinnis Valenciennes, 1836 et Mugil cephalus Linnaeus, 1758 dans les complexes lagunaires Ebrié et Grand-Lahou, Côte d'Ivoire. Thèse unique de l'Université Félix Houphouët-Boigny, Abidjan (Côte d'Ivoire), p. 195.

Elmanama AA, Afifi S, Bahr S. 2006. Seasonal and spatial variation in the monitoring parameters of gaza beach during 2002-2003. Environ Res., 25: 25-33. DOI : http://doi.org/10.1016/ j.envres.2005.07.005

Hartel P, Rodgers K, Fisher JA, McDonald JL, Gentit LC, Otero E, Rivera-Torres Y, Bryant TL, Jones SH. 2005. Survival and regrowth of fecal enterococci in desiccated and rewetted sediments. Proceedings of the 2005 Georgia Water Resources Conference. University of Georgia, Athens, Georgia, U.S.A.

Inza B, Métongo BS, Assoi OE, Albert T, Yobou B. 2009. Caractérisation physico-chimique des eaux et des sédiments de surface de la baie des milliardaires, lagune Ebrié, Côte d'Ivoire. Rev. Ivoir. Sci. Technol., 13: 135-154.

Ishii S, Ksoll WB, Hicks RE, Sadowsky MJ. 2006. Presence and growth of naturalized Escherichia coli in temperate soils from Lake Superior watersheds. Applied Environmental 
Microbiology, 72(1): 612-62. DOI : http://dx.doi.org/10.1128/AEM.72.1.61 2-621.2006

Issola Y, Kouassi AM, Dongui BK, Biemi J. 2008. Caractérisation physicochimiques d'une lagune côtière tropicale : lagune de Fresco (Côte d'Ivoire). Afrique Sciences, 04(3): 368393.

Kambiré O, Adingra AA, Eblin SG, AKA N, Kakou AC, Koffi-Nevry R. 2014. Caractérisation des eaux d'une lagune estuarienne de la Côte d'Ivoire : la lagune Aby. Larhyss Journal, 20: 95110.

Kambiré O, Adingra AA, Kakou AC, KoffiNévry R. 2012. Indicateurs de pollution fécale dans une lagune tropicale à forte influence continentale (Lagune Aby, Côte d'Ivoire). Agronomie Africaine, 24(2): 89-100.

Kon T, Weir SC, Trevors JT, Lee H, Champagne J, Meunier L, Brousseau R, Masson L. 2007. Microarray analysis of Escherichia coli strains from interstitial beach waters of Lake Huron (Canada). Applied Environment Microbiology, 73(23): 7757-7758. DOI : $\quad$ http://dx.doi.org/10.1128/ AEM.01333-07

Koné B, Doumbia M, Adji FX. 2007. Approche écosystémique à la gestion des maladies diarrhéiques en milieu périurbain : cas d'un village lagunaire dans la commune de Yopougon (Abidjan, Côte d'Ivoire). Projet de recherche Centre Suisse de Recherches Scientifiques en Côte d'Ivoire, rapport final, p. 81.

Kouassi AM. 2005. Hydrochimie et qualité des eaux de deux lagunes tropicales de Côte d'Ivoire (Ebrié, Grand Lahou). Thèse de Doctorat, Université de Cocody. Abidjan (Côte d'Ivoire), p. 147.

Lwamba BJ, Katim MAMA, Kiwaya AT, Ipungu LR, Nyongombe UN. 2015. Variations de la température de l'eau des étangs en période froide à
Lubumbashi (R. D. Congo) et implications pour la production des poissons. J. Appl. Biosci., 90: 84298437. DOI : http://dx.doi.org/10.4314/ jab.v90i1.5

OMS. 2004. Guidelines for Drinking Water Quality, (vol 1; 3rd edn). World Health Organization (WHO): Genève, Suisse, p. 595.

Traoré A, Soro G, Kouadio KE, Bamba SB, Oga MS, Soro N, Biémi J. 2012. Evaluation des paramètres physiques, chimiques et bactériologiques des eaux d'une lagune tropicale en période d'étiage : la lagune Aghien (Côte d'Ivoire). Int. J. Biol. Chem. Sci., 6(6): 7048-7058.

DOI: http://dx.doi.org/10.4314/ijbcs.v6i6.40

Tuo AD, Yeo KM, Soro MB, Trokourey A, Bokra Y. 2013. Contamination by nutrientsand heavy metals in the Ebrie Lagoon (Abidjan, Ivory Coast). Res. J. Environ. Toxicol., 6: 198-209. DOI : http://dx.doi.org/10.3923/ijct.2013.10.2 1

Vezzulli L, Pezzati E, Repetto B, Stauder M, Giusto G, Pruzzo C. 2008. A general role for surface membrane proteins in attachment to chitin particles and copepods of environmental and clinical Vibrios. Lett. App. Microbiol., 46: 119125. DOI: http://dx.doi.org/10.1128/ AEM.03811-13

Whitman RL, Nevers MB. 2003. Foreshore sand as a source of Escherichia coli in nearshore water of a Lake Michigan beach. Appled Environmental Microbiology, 69(9): 5555-5562. DOI: http://dx.doi.org/10.1128/AEM.69.9.55 55-5562.2003 